\title{
The diffusion-driven instability and complexity for a single-handed discrete Fisher equation
}

\author{
Guang Zhang*and Ruixuan Zhang \\ School of Science, Tianjin University of Commerce \\ Tianjin 300134, P. R. China \\ Yubin Yan \\ Department of Mathematical and Phisical Science, Faculty of Science \& Engineering \\ University of Chester \\ Thornton Science Park, Pool Lane, Ince, Chester, CH24NU, UK
}

\begin{abstract}
For a reaction diffusion system, it is well known that the diffusion coefficient of the inhibitor must be bigger than that of the activator when the Turing instability is considered. However, the diffusion-driven instability/Turing instability for a single-handed discrete Fisher equation with the Neumann boundary conditions may occur and a series of 2-periodic patterns have been observed. Motivated by these pattern formations, the existence of 2-periodic solutions is established. Naturally, the periodic double and the chaos phenomenon should be considered. To this end, a simplest two elements system will be further discussed, the flip bifurcation theorem will be obtained by computing the center manifold, and the bifurcation diagrams will be simulated by using the shooting method. It proves that the Turing instability and the complexity of dynamical behaviors can be completely driven by the diffusion term. Additionally, those effective methods of numerical simulations are valid for experiments of other patterns, thus, are also beneficial for some application scientists.
\end{abstract}

MSC(2010): 39A10.

Keywords: Discrete Fisher equation, Turing instability, Turing bifucation, flip bifurcation, shooting method.

\section{Introduction}

Turing structures arise when imbalances in diffusion rates make a stable steadystate system sensitive to small heterogeneous perturbations. The ideas have profoundly influenced theoretical understanding of pattern formation in nonlinear

*Corresponding authors. E-mail address: lxyzhg@tjcu.edu.cn (G. Zhang) 
optics (see [1], [25] and [49]), fluid dynamic (see [7] and [29]), colloidal lithography [46], epidemiology (see [35] and [40]), cell biology [17], solid-liquid-vapour system [31], biological morphogenesis (see [44], [9], [16], [38] and [43]), nanostructure (see [4], [13], [19] and [41]), biochemical networks [42], phytoplankton dynamics [21], material science (see [5], [13], [39] and [6]), electrochemistry [23], chemical reaction (see [44], [32], [20], [19] and [41]), social science [2], etc.

A reaction-diffusion system is said to exhibit Turing or the diffusion-driven instability if the steady state solution is stable to small spatial perturbations in absence of diffusion, but unstable when diffusion is present. Turing structures typically emerge in reaction diffusion processes far from thermodynamic equilibrium [44], in which the diffusion coefficient of the inhibitor must be larger than that of the activator. That is, a reaction diffusion equation can not lead to the Turing instability. For example, the Fisher equation

$$
\frac{\partial u}{\partial t}=D \Delta u+u(a-b u), D, a, b>0,
$$

is well known and has been studied by many authors since it is a basic reaction diffusion equation which can be used to model population dynamics with migration, temperature distribution under control, chemical reactions, etc. When $D=0$, equation (1) is reduced to the logistic equation

$$
\frac{d x(t)}{d t}=x(t)(a-b x(t)),
$$

which has a stable positive steady state $a / b$. We note that $u^{*}(t, x) \equiv a / b$ is also the positive steady state of (1) with the Neumann or periodic boundary conditions, and clearly, it is also stable.

Equation (2) can be rewritten by $((d x(t)) /(x(t) d t))=a-b x(t)$. Thus, the constant $a>0$ is the birth rate, $b x(t)$ is the death rate, and $b$ is a positive constant. When the random walk is added to Equation (2), the Fisher equation (1) will be obtained.

Many authors have argued that the discrete time models governed by $\mathrm{d}$ ifference equations are more appropriate than the continuous ones when the populations have nonoverlapping generations, for example, see Freedman [8], Murry [30], Punithan et al. [33], Huang et al. [15] and Wang et al. [45]. By using the different discrete methods, from equation (2) we can obtain the discrete logistic equations:

$$
\begin{gathered}
x_{t+1}=r x_{t}\left(1-x_{t}\right), \\
x_{t+1}=x_{t} \exp \left(a-b x_{t}\right),
\end{gathered}
$$

or

$$
x_{t+1}=\frac{e^{a h} x_{t}}{1+\left(\left(e^{a h}-1\right) b / a\right) x_{t}},
$$

see Liu and Cui [24], where $h>0$ is the discrete step size. The dynamics of (3) and (4) can become "chaotic" for certain parameter values while their "motherversion" (2) has very simple dynamics, see May [26]. It is elementary to note 
that the fixed points of (5) are 0 and $a / b$ as they are in the corresponding continuous case (2). Also $x_{t} \rightarrow a / b$ monotonously as $t \rightarrow \infty$ when $x_{0}>0$. This is exactly the same as in (2). Thus, (5) can be considered as a better discretization of the continuous logistic equation.

Let

$$
p=e^{a h} \text { and } y_{t}=\left(\left(e^{a h}-1\right) b / a\right) x_{t} .
$$

From (5), we have

$$
y_{t+1}=\frac{p y_{t}}{1+y_{t}}
$$

where $p>1$. Clearly, equation (6) has the steady states 0 and $p-1$, the zero solution is not stable, and the positive steady state $p-1$ is stable.

In almost any real ecosystem, the population dynamics include both temporal reproduction processes and spatial diffusion processes, see Punithan et al. [33]. Let $Z^{+}=\{0,1,2, \cdots\}$. For any $k, l \in Z^{+}$with $k<l$, denote $[k, l]=\{k, k+1, \cdots, l\}$, and consider the discrete reaction diffusion equation of the form

$$
u_{i j}^{t+1}=d \Delta u_{i j}^{t}+\frac{p u_{i j}^{t}}{1+u_{i j}^{t}},(i, j) \in[1, m] \times[1, n],
$$

with the Neumann boundary conditions

$$
\left\{\begin{array}{c}
u_{i, 0}^{t}=u_{i, 1}^{t}, u_{i, n}^{t}=u_{i, n+1}^{t}, i \in[0, m+1], \\
u_{0, j}^{t}=u_{1, j}^{t}, u_{m, j}^{t}=u_{m+1, j}^{t}, j \in[0, n+1],
\end{array}\right.
$$

for $t \in Z^{+}$, where $m$ and $n$ are positive integers, $d>0$ is the diffusion coefficient, $p>1$, and

$$
\Delta u_{i j}^{t}=u_{i+1, j}^{t}+u_{i, j+1}^{t}+u_{i-1, j}^{t}+u_{i, j-1}^{t}-4 u_{i j}^{t} .
$$

Now, we have a problem. Can the diffusion term of (7)-(8) drive instability? That is, can (7)-(8) be the diffusion-driven instability or Turing instability? Indeed, there is the different statement for the space- and time-discrete model, the dynamical behaviors of activator and inhibitor from $t$ to $t+1$ contains two distinctly different processes, one is the "reaction" stage, the other is "dispersal" stage, for example, see Mistro et al. [34] and [28], Punithan et al. [33], Huang et al. [14] and [15] for the predator-prey model, competitive system ([12] and [22]), diffusion-migeration systems [51], statistical physics [3], Gierer-Meinhardt system [45] and so on. However, all above models are the space- and timediscrete systems.

$\mathrm{Xu}$ et al. [48] and [47] considered a two-dimensional logistic coupled map lattice of the form

$$
u_{i j}^{t+1}=(1-\varepsilon) f\left(u_{i j}^{t}\right)+\frac{\varepsilon}{4}\left[f\left(u_{i+1, j}^{t}\right)+f\left(u_{i, j+1}^{t}\right)+f\left(u_{i-1, j}^{t}\right)+f\left(u_{i, j-1}^{t}\right)\right]
$$

or

$$
u_{i j}^{t+1}=f\left(u_{i j}^{t}\right)+\varepsilon \Delta f\left(u_{i j}^{t}\right)
$$


with the periodic boundary conditions

$$
u_{0, j}^{t}=u_{m, j}^{t}, u_{1, j}^{t}=u_{m+1, j}^{t}, u_{i, 0}^{t}=u_{i, n}^{t}, u_{i, 1}^{t}=u_{i, n+1}^{t},
$$

where $f(x)=\lambda x(1-x)$ for $\lambda \in(1,3)$, and

$$
\Delta f\left(u_{i j}^{t}\right)=f\left(u_{i+1, j}^{t}\right)+f\left(u_{i, j+1}^{t}\right)+f\left(u_{i-1, j}^{t}\right)+f\left(u_{i, j-1}^{t}\right)-4 f\left(u_{i j}^{t}\right) .
$$

The conditions of Turing instability had been obtained and some interest patterns had also been simulated in [48] and [47].

The present paper is motivated by $\mathrm{Xu}$ et al. [48] and [47] and the problem (7)-(8) will be considered. Note that the diffusion term in (7) is linear and that the positive steady state $p-1$ of (6) is stable for all $p>1$. These will cause some difficulties in numerical simulations. We simulate all possible cases, however, only obtain the 2 -periodic patterns in this paper. In this case, we consider the different problems from Xu et al. [48] and [47], for example, the existence of 2-periodic solutions, the flip bifurcation theorem and the bifurcation diagrams of a simplest two elements system. In particular, for the simplest two elements system, the other periodic orbits are also obtained by using the numerical simulation. Thus, the Turing instability and the complexity of dynamical behaviors are completely driven by the diffusion term.

The present paper will be organized as follows. The necessary and sufficient conditions of Turing instability for problem (7)-(8) will be established in the following section. To date most numerical simulations of reaction-diffusion models have used small random perturbations about the kinetic steady state as initial data. However, in view of the discrete Grobman-Hartman Theorem [18], we find that the numerical simulations are obvious effected by using the linear combinations of the eigenvectors. By using such method, some Turing patterns will be simulated in Section 3. The 2-periodic patterns have been observed. Thus, in Section 4, the existence of 2-periodic solutions will be proved by using the inverse function theorem. Naturally, we should also further discuss the periodic double and the chaos phenomenon of (7)-(8). Unfortunately, all patterns are 2-periodic. To obtain other dynamical informations of (7)-(8), we will further analyse a simplest two elements system when $p>1$ is fixed and $d>0$ is a parameter. As a result, we find that this system can cause the complexity. These facts will be given in Section 5. In the final section, we will conclude some conclusions and give some open problems.

\section{Linearization analysis and the Turing instabil- ity}

The idea of linearization analysis is an efficient approach. It has been also adopted in the work [36]. Note that $u_{i j}^{t} \equiv p-1$ is a solution of (7)-(8). Thus, we can obtain the linearization problem of (7)-(8) about the kinetic steady state

$$
v_{i j}^{t+1}=d \Delta v_{i j}^{t}+\frac{1}{p} v_{i j}^{t}, t=0,1,2, \cdots,
$$


with the Neumann boundary conditions

$$
\left\{\begin{array}{c}
v_{i, 0}^{t}=v_{i, 1}^{t}, v_{i, n}^{t}=v_{i, n+1}^{t}, i \in[0, m+1], \\
v_{0, j}^{t}=v_{1, j}^{t}, v_{m, j}^{t}=v_{m+1, j}^{t}, j \in[0, n+1] .
\end{array}\right.
$$

The eigenvalues and eigenvectors of the discrete Neumann equations are demonstrated in the Appemdix.

Assume that $\left\{v_{i j}^{t}\right\}$ is a solution of (10)-(11). According to Able lemma and the corresponding boundary condition (11), we have

$$
\begin{aligned}
\sum_{i=1}^{m} \sum_{j=1}^{n} \varphi_{i j}^{(k l)} v_{i j}^{t+1} & =d \sum_{i=1}^{m} \sum_{j=1}^{n} \varphi_{i j}^{(k l)} \Delta v_{i j}^{t}+\frac{1}{p} \sum_{i=1}^{m} \sum_{j=1}^{n} \varphi_{i j}^{(k l)} v_{i j}^{t} \\
& =d \sum_{i=1}^{m} \sum_{j=1}^{n} v_{i j}^{t} \Delta \varphi_{i j}^{(k l)}+\frac{1}{p} \sum_{i=1}^{m} \sum_{j=1}^{n} \varphi_{i j}^{(k l)} v_{i j}^{t} \\
& =\left(\frac{1}{p}-d \lambda_{k l}\right) \sum_{i=1}^{m} \sum_{j=1}^{n} \varphi_{i j}^{(k l)} v_{i j}^{t} .
\end{aligned}
$$

Let

$$
V^{t}=\sum_{i=1}^{m} \sum_{j=1}^{n} \varphi_{i j}^{(k l)} v_{i j}^{t}
$$

We get that

$$
V^{t+1}=\left(\frac{1}{p}-d \lambda_{k l}\right) V^{t}, t=0,1,2, \cdots
$$

If $\left\{V^{t}\right\}$ is a solution of (12), then $v_{i j}=V^{t} \varphi_{i j}^{(k l)}$, is clearly a solution of (10)-(11). Thus, we have the following result.

Theorem 1. (10)-(11) is stable if and only if equation (12) is stable for all $(k, l) \in[1, m] \times[1, n]$.

Note that the eigenvalue of (12) is $d \lambda_{k l}-1 / p$, in view of Theorem 1 , we immediately obtain the following result.

Corollary 1. (10)-(11) is stable if and only if the inequality

$$
\left|\frac{1}{p}-d \lambda_{k l}\right|<1
$$

holds for all $(k, l) \in[1, m] \times[1, n]$.

Corollary 2. (10)-(11) is stable if and only if the inequality

$$
\lambda_{m n}=4\left[\cos ^{2} \frac{\pi}{2 m}+\cos ^{2} \frac{\pi}{2 n}\right]<\frac{1}{d}+\frac{1}{d p}
$$

holds. 
Proof. We know that the inequality

$$
\left|\frac{1}{p}-d \lambda_{k l}\right|<1
$$

holds if and only if the inequality

$$
-1<\frac{1}{p}-d \lambda_{k l}<1
$$

or

$$
\frac{1}{p}-1<d \lambda_{k l}<1+\frac{1}{p}
$$

holds. Note that $p>1$ which implies that the first one of the above inequalities is obvious. On the other hand,

$$
\begin{aligned}
\max _{k \in[1, m], l \in[1, n]}\left\{\lambda_{k l}\right\} & =4 \max _{i \in[1, m], j \in[1, n]}\left[\sin ^{2} \frac{(k-1) \pi}{2 m}+\sin ^{2} \frac{(s-1) \pi}{2 n}\right] \\
& =4\left[\sin ^{2} \frac{(m-1) \pi}{2 m}+\sin ^{2} \frac{(n-1) \pi}{2 n}\right] \\
& =4\left[\cos ^{2} \frac{\pi}{2 m}+\cos ^{2} \frac{\pi}{2 n}\right] .
\end{aligned}
$$

The proof is complete.

Corollary 3. (10)-(11) is unstable if and only if there exists $(k, l) \in[1, m] \times$ $[1, n]$ such that

$$
\lambda_{k l}>\frac{1}{d}+\frac{1}{d p}
$$

holds. That is, the positive steady state solution $u_{i j}^{t} \equiv p-1$ of (7)-(8) is Turing unstable if and only if there exists $(k, l) \in[1, m] \times[1, n]$ such that inequality (15) holds.

\section{Turing patterns}

In this section, we will give some numerical simulations when all conditions of the Turing instability hold. To achieve the desired results of numerical simulation, we need to give some theoretical analysis. They will be obtained in the following subsection. An example will be given in the second subsection and a series of 2-periodic patterns can be observed.

\subsection{Some theoretical analysis}

Consider now a recurrence sequence defined by

$$
x_{t+1}=f\left(x_{t}\right) \text { for } x_{t} \in R^{n},
$$


where $f$ is sufficiently smooth, $f(0)=0$. Let the eigenvalues of the Jacobian matrix $A$ evaluated at the fixed point $x_{0}=0$ be $\mu_{1}, \mu_{2}, \cdots, \mu_{n}$. Assume that there are $n_{+}$multipliers outside the unit circle, $n_{0}$ multipliers on the unit circle, and $n_{-}$multipliers inside the unit circle. The fixed point 0 is called to be hyperbolic if $n_{+}+n_{-}=n$. The following lemma is Theorem 2.4 in [18].

Lemma 1. The phase portraits of (16) near two hyperbolic fixed points, $x_{0}$ and $y_{0}$, are locally topologically equivalent if and only if these fixed points have the same number $n_{-}$and $n_{+}$of multipliers with $|\mu|<1$ and $|\mu|>1$, respectively, and the signs of the products of all the multipliers with $|\mu|<1$ and with $|\mu|>1$ are the same for both fixed points.

In view of Lemma 1, suppose that the equilibrium is hyperbolic and there are $n_{+}$multipliers outside the unit circle, and $n_{-}$multipliers inside the unit circle. Using an eigenbasis, we can also rewrite the system (16) as

$$
\left\{\begin{array}{c}
u_{t+1}=B u_{t}+g\left(u_{t}, v_{t}\right), \\
v_{t+1}=C v_{t}
\end{array}\right.
$$

where $u_{t} \in R^{n_{+}}, v_{t} \in R^{n_{-}}$, the eigenvalues of $B \in R^{n_{+}} \times R^{n_{+}}$are outside the unit circle, and the eigenvalues of $C \in R^{n_{-}} \times R^{n_{-}}$are inside the unit circle.

Note that $\lim _{t \rightarrow \infty} v_{t}=0$, in view of Lemma 1 , we find that the long-time dynamical behavior of (16) should depend on the system

$$
u_{t+1} \approx B u_{t}+g\left(u_{t}, 0\right) .
$$

Thus, the initial values should be chosen from the unstable tangent bundles.

Remark 1. The numerical simulations of patterns are difficult for the random initial values when the number of $|\mu|>1$ is small. For problem (7)-(8), we assume that there exists $(k, l) \in[1, m] \times[1, n]$ such that

$$
\lambda_{k l}>\frac{1}{d}+\frac{1}{d p}
$$

which implies that

$$
\lambda_{k+\tau, l+\iota}>\frac{1}{d}+\frac{1}{d p} \text { for }(\tau, \iota) \in[0, m-k] \times[0, n-l] .
$$

Thus, the initial values should be chosen by

$$
u_{i j}^{0}=p-1+\varepsilon \sum_{\tau=0}^{m-k} \sum_{\iota=0}^{n-l} c_{\tau \iota} \varphi_{i j}^{(k+\tau, l+\iota)} .
$$

In particular, we assume that

$$
\lambda_{m n}>\frac{1}{d}+\frac{1}{d p} \text { and } \max \left\{\lambda_{m-1, n}, \lambda_{m, n-1}\right\}<\frac{1}{d}+\frac{1}{d p}
$$


hold. Then, the initial values should be chosen by

$$
u_{i j}^{0}=p-1+\varepsilon \varphi_{i j}^{(m n)} .
$$

In this case, if we randomly choose the initial values $\tau_{i j}^{0} \in[-1,1]$ for $(i, j) \in$ $[1, m] \times[1, n]$, then there exists $c_{k l}$ such that

$$
\tau_{i j}^{0}=\sum_{i=1}^{m} \sum_{j=1}^{n} c_{k l} \varphi_{i j}^{(k l)} \text { for }(i, j) \in[1, m] \times[1, n] .
$$

Let

$$
u_{i j}^{0}=p-1+\varepsilon \sum_{i=1}^{m} \sum_{j=1}^{n} c_{k l} \varphi_{i j}^{(k l)} .
$$

It is very difficult to achieve the desired results of numerical simulation. Indeed, Lemma 1 implies that

$$
\lim _{t \rightarrow \infty} u_{i j}^{t}=p-1 \text { for }(i, j) \in[1, m] \times[1, n]
$$

when $c_{m n}=0$. In fact, the probability of $c_{m n} \neq 0$ is $1 / m n$.

Remark 2. Turing patterns of numerical simulation will depend on the tangent eigenvectors of maximum norm eigenvalues. For any $v_{i j}^{0} \in R$ with $(i, j) \in[1, m] \times[1, n]$, then, there exists $c_{k l} \in R$ such that

$$
v_{i j}^{0}=\sum_{k=1}^{m} \sum_{l=1}^{n} c_{k l} \varphi_{i j}^{(k l)} \text { for }(i, j) \in[1, m] \times[1, n] .
$$

From (10)-(11), we have

$$
\begin{aligned}
v_{i j}^{1} & =d \Delta v_{i j}^{0}+\frac{1}{p} v_{i j}^{0} \\
& =d \Delta\left(\sum_{k=1}^{m} \sum_{l=1}^{n} c_{k l} \varphi_{i j}^{(k l)}\right)+\frac{1}{p} \sum_{k=1}^{m} \sum_{l=1}^{n} c_{k l} \varphi_{i j}^{(k l)} \\
& =d \sum_{k=1}^{m} \sum_{l=1}^{n} c_{k l} \Delta \varphi_{i j}^{(k l)}+\frac{1}{p} \sum_{k=1}^{m} \sum_{l=1}^{n} c_{k l} \varphi_{i j}^{(k l)} \\
& =\sum_{k=1}^{m} \sum_{l=1}^{n} c_{k l}\left(\frac{1}{p}-d \lambda_{k l}\right) \varphi_{i j}^{(k l)} \\
& v_{i j}^{2}=d \Delta v_{i j}^{1}+\frac{1}{p} v_{i j}^{1} \\
& =\sum_{k=1}^{m} \sum_{l=1}^{n} c_{k l}\left(\frac{1}{p}-d \lambda_{k l}\right)^{2} \varphi_{i j}^{(k l)}
\end{aligned}
$$




$$
v_{i j}^{t}=\sum_{k=1}^{m} \sum_{l=1}^{n} c_{k l}\left(\frac{1}{p}-d \lambda_{k l}\right)^{t} \varphi_{i j}^{(k l)} \text { for }(i, j) \in[1, m] \times[1, n] .
$$

Let

$$
\left(m^{\prime}, n^{\prime}\right)=\left\{(k, l): \max _{c_{k l} \neq 0}\left|\frac{1}{p}-d \lambda_{k l}\right|\right\} .
$$

In this case, we have

$$
v_{i j}^{t}=\sum_{k=1}^{m^{\prime}} \sum_{l=1}^{n^{\prime}} c_{k l}\left(\frac{1}{p}-d \lambda_{k l}\right)^{t} \varphi_{i j}^{(k l)}
$$

or

$$
\begin{aligned}
\frac{v_{i j}^{t}}{\left(\frac{1}{p}-d \lambda_{m^{\prime} n^{\prime}}\right)^{t}} & =\sum_{k=1}^{m^{\prime}} \sum_{l=1}^{n^{\prime}} c_{k l}\left(\frac{\frac{1}{p}-d \lambda_{k l}}{\frac{1}{p}-d \lambda_{m^{\prime} n^{\prime}}}\right)^{t} \varphi_{i j}^{(k l)} \\
& \sim c_{m^{\prime} n^{\prime}} \varphi_{i j}^{(k l)} \text { for }(i, j) \in[1, m] \times[1, n] .
\end{aligned}
$$

In view of Lemma 1 and (23), Turing patterns of numerical simulation will depend on the tangent eigenvector of maximum norm eigenvalue. That is, the tangent eigenvector of maximum norm eigenvalue will be the embryo of the Turing patterns. These facts will also be seen in the following numerical simulations.

\subsection{An example}

In this subsection, we assume that $p=2$ and $m=n=64$. In this case, we have

$$
\begin{gathered}
\lambda_{k l}=4\left[\sin ^{2} \frac{(k-1) \pi}{2 \times 64}+\sin ^{2} \frac{(l-1) \pi}{2 \times 64}\right], \\
\lambda_{64,64}=8 \sin ^{2} \frac{(64-1) \pi}{2 \times 64} \approx 7.9952
\end{gathered}
$$

and

$$
\lambda_{63,64}=\lambda_{64,63}=4\left[\sin ^{2} \frac{(64-1) \pi}{2 \times 64}+\sin ^{2} \frac{(64-2) \pi}{2 \times 64}\right] \approx 7.9880 .
$$

Suppose that

$$
7.9880<\frac{1}{d}+\frac{1}{2 d}=\frac{3}{2 d}<7.9952
$$

or

$$
0.18761 \approx \frac{3}{2 \times 7.9952}<d<\frac{3}{2 \times 7.9880} \approx 0.18778 \text {. }
$$




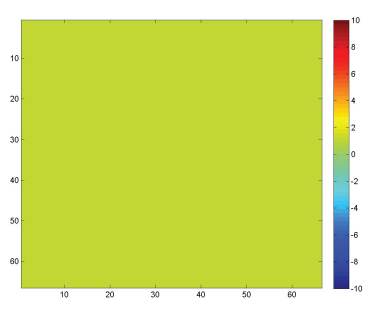

(a) $t=0$

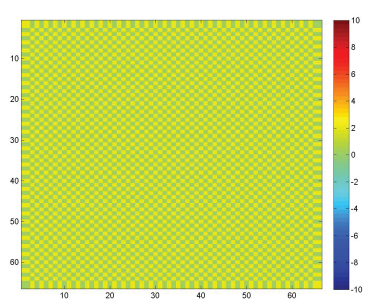

(c) $t=90$

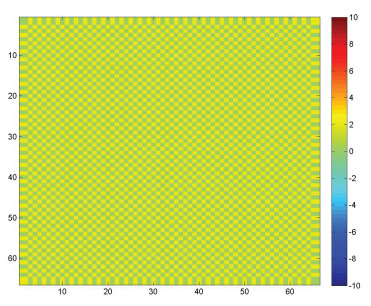

(b) $t=89$

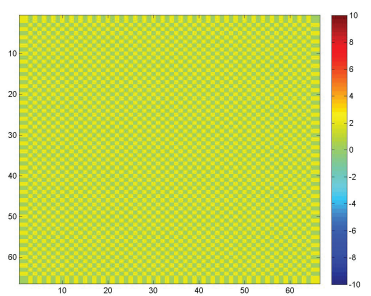

(d) $t=200000$

Figure 1: $m=n=64, p=2, d=50000$ and $u_{i j}^{0}$ is chosen by (30).

Then, the condition (20) holds. In this case, we choose that $d=0.1877$ and that

$$
u_{i j}^{0}=1+0.01 \cos \frac{(64-1)(2 i-1) \pi}{2 \times 64} \cos \frac{(64-1)(2 j-1) \pi}{2 \times 64},
$$

an eventual 2-periodic pattern is obtained.

On the other hand, we also choose the initial values distribution (24) and give a series of numerical simulations for $d>0.18761$. Similarly, all patterns are eventually 2 -periodic. The only difference is the time from the initial values distribution to the 2-periodic patterns. For example, for $d=50000$, see Figure 1. From these simulations, we see that the patterns are two period mode from $t=1$, that is, all patterns are selfsaue when $t$ is odd and they are also same if $t>0$ is even, for example, see (c) and (d) in Figure 1 they are obtained for $t=90$ and 200000, respectively. In fact, (a) in Figure 1 has been seen the embryo (d) in Figure 1.

Let

$$
d>\frac{3}{2 \times 4 \sin ^{2} \frac{\pi}{2 \times 64}} \approx 622.64 .
$$

In this case, we can choose the initial values distribution

$$
u_{i j}^{0}=1+0.01 \cos \frac{(k-1)(2 i-1) \pi}{2 \times 64} \cos \frac{(l-1)(2 j-1) \pi}{2 \times 64}
$$

for $(k, l) \in[1,64] \times[2,64]$ or $(k, l) \in[2,64] \times[1,64]$. The obtained patterns are also 2-periodic. For example, we fix $d=50000$ and choose different $k$ and 


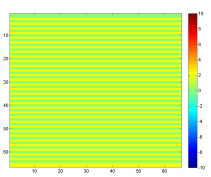

(a) $k=1$ and $l=2$

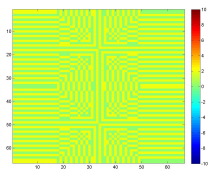

(e) $k=3$ and $l=2$

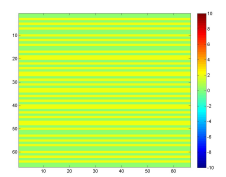

(b) $k=1$ and $l=19$

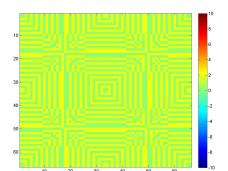

(f) $k=3$ and $l=3$

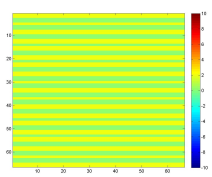

(c) $k=1$ and $l=37$

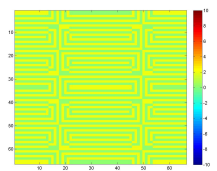

(g) $k=3$ and $l=7$

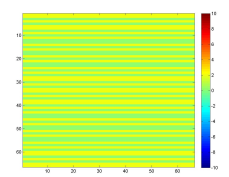

(d) $k=1$ and $l=53$

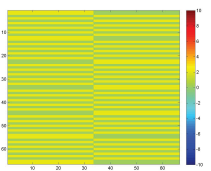

(h) $k=2$ and $l=59$

Figure 2: $m=n=64, p=2, d=50000$ and $u_{i j}^{0}$ is chosen by (31).

$l$, the patterns are obtained in Figure 2 in which we can obtain the following facts: (i) all patterns are the alternation of two different forms, (ii) for the fixed $k=1$ and the different $l$, there are the difference of the widths of the green or yellow stripes, see (a)-(d), and (iii) the area $[1,64] \times[1,64]$ will be cut apart the corresponding parts which depend on the selections of $k$ and $l$, see (e)-(h).

For $(k, l) \in[1,64] \times[2,64]$ or $(k, l) \in[2,64] \times[1,64]$, some eigenvalues are same, however, their corresponding eigenvectors are different. For example, the minimal eigenvalue is

$$
\lambda_{2,1}=\lambda_{1,2}=4 \sin ^{2} \frac{\pi}{2 \times 64} \approx 2.4091 \times 10^{-3}
$$

which have respectively the corresponding eigenvectors

$$
\cos \frac{(2 i-1) \pi}{2 \times 64} \text { for } i \in[1,64]
$$

and

$$
\cos \frac{(2 j-1) \pi}{2 \times 64} \text { for } j \in[1,64] .
$$

In this case, we choose the initial values distributions

$$
\begin{aligned}
& u_{i j}^{0}=1+0.01 \cos \frac{(2 i-1) \pi}{2 \times 64}+0.01 \cos \frac{(2 j-1) \pi}{2 \times 64}, \\
& u_{i j}^{0}=1+0.01 \cos \frac{(2 i-1) \pi}{2 \times 64}+0.005 \cos \frac{(2 j-1) \pi}{2 \times 64}
\end{aligned}
$$

and

$$
u_{i j}^{0}=1+0.01 \cos \frac{(2 i-1) \pi}{2 \times 64}+0.09 \cos \frac{(2 j-1) \pi}{2 \times 64}
$$

and the different 2-periodic patterns can be obtained, please see the corresponding (a), (b) and (c) in Figure 3. For $d=50000, m=n=64$, and $p=2$, we can 


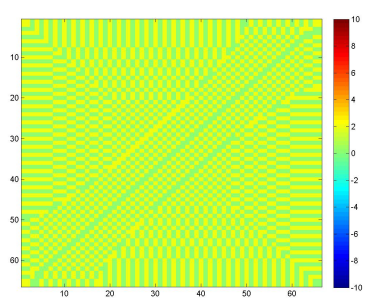

(a) $u^{0}$ is $(32)$

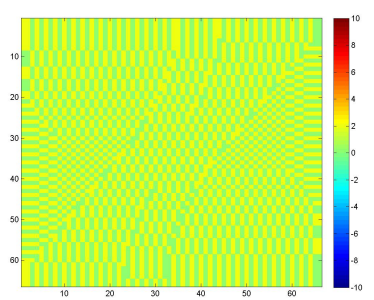

(c) $u^{0}$ is $(34)$

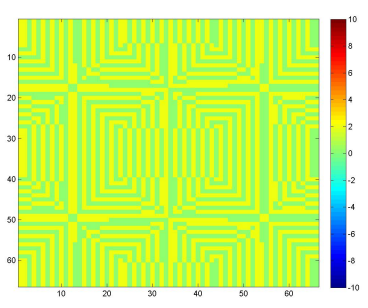

(b) $u^{0}$ is $(33)$

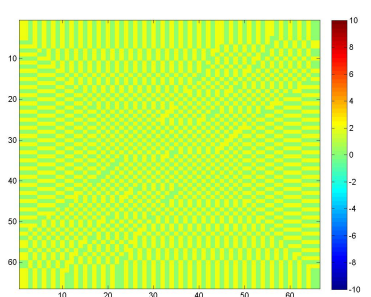

(d) $u^{0}$ is $(35)$

Figure 3: $m=n=64, p=2, d=50000$.

further choose the initial values distribution

$$
\begin{aligned}
u_{i j}^{0}= & 1+0.01 \cos \frac{(2 j-1) \pi}{2 \times 64} \\
& +0.01 \cos \frac{(3-1)(2 i-1) \pi}{2 \times 64} \cos \frac{(4-1)(2 j-1) \pi}{2 \times 64} .
\end{aligned}
$$

The simulation result is the pattern (d) in Figure 3, the eigenvector of the greater eigenvalue is the leader. The pattern is exactly the same as one of the initial values distribution

$$
u_{i j}^{0}=1+0.01 \cos \frac{(3-1)(2 i-1) \pi}{2 \times 64} \cos \frac{(4-1)(2 j-1) \pi}{2 \times 64} .
$$

Thus, (23) holds true.

From the above pattern formations, they are the flip bifurcation, the periodic double, and the chaos phenomenon will occur for problem (7)-(8). Thus, we hope to simulate the other different periodic patterns, unfortunately, we fail.

\section{The existence of 2-periodic solutions}

From previous discussion, we found that problem (7)-(8) should have many 2periodic solutions when some conditions hold. In this section, we will further 
discuss such problem. To this end, we consider the nonlinear algebraic system of the form

$$
\left\{\begin{array}{c}
u_{i j}^{1}=d \Delta u_{i j}^{0}+\frac{p u_{i j}^{0}}{1+u_{i j}^{0}},(i, j) \in[1, m] \times[1, n], \\
u_{i j}^{0}=d \Delta u_{i j}^{1}+\frac{p u_{i j}^{i}}{1+u_{i j}^{t}},(i, j) \in[1, m] \times[1, n], \\
u_{i, 0}^{t}=u_{i, 1}^{t}, u_{i, n}^{t}=u_{i, n+1}^{t}, i \in[0, m+1], t=0,1 \\
u_{0, j}^{t}=u_{1, j}^{t}, u_{m, j}^{t}=u_{m+1, j}^{t}, j \in[0, n+1], t=0,1 .
\end{array}\right.
$$

We need the following inverse function theorem which can be seen Theorem 1.2 .1 in $[10]$.

Lemma 2. Let $G$ be a $C^{r}$ map from an open set $W$ into $R^{m}$, with $r \geq 1$. Let $x$ be a point of $W$ and assume that the Jacobian matrix $D G(x)$ is invertible. Then $x$ has an open neighborhood $U$ such that the restriction of $G$ to $U$ is a $C^{r}$ diffeomorphism onto the open set $G(U)$.

Theorem 2. For all $(k, l) \in[1, m] \times[1, n]$, assume that

$$
\frac{1}{d p} \neq \lambda_{k l} \neq \frac{p}{d}
$$

hold. Then system (30) has a nontrivial solution $\left(u_{*}^{0}, u_{*}^{1}\right) \in U \times V$, where $U \times V$ is an open neighborhood of $\left(u^{0}, u^{1}\right), u^{0}=\left(u_{i j}^{0}\right)_{m \times n}, u^{1}=\left(u_{i j}^{1}\right)_{m \times n}$, and $u_{i j}^{0}$ and $u_{i j}^{1}$ are 0 or $p-1$ for $[1, m] \times[1, n]$. In particular, when the inequality

$$
\lambda_{m n}>\frac{1}{d}+\frac{1}{d p}
$$

holds and

$$
\frac{1}{d p} \neq \lambda_{k l} \text { for }(k, l) \in[1, m] \times[1, n],
$$

system (30) has a nontrivial positive solution $\left(u_{*}^{0}, u_{*}^{1}\right) \in U \times V$, where $U \times V$ is an open neighborhood of $\left(u^{0}, u^{1}\right), u^{0}=\left(u_{i j}^{0}\right)_{m \times n}, u^{1}=\left(u_{i j}^{1}\right)_{m \times n}$, and $u_{i j}^{0}=u_{i j}^{1}=p-1$ for $[1, m] \times[1, n]$.

Proof. Note that

$$
\begin{gathered}
\left(\frac{p u}{1+u}\right)^{\prime}=\frac{p}{(1+u)^{2}}, \\
\left(\frac{p u}{1+u}\right)^{\prime}{ }_{u=0}=p
\end{gathered}
$$

and

$$
\left.\left(\frac{p u}{1+u}\right)^{\prime}\right|_{u=p-1}=\frac{1}{p}
$$


Thus, the Jacobian matrix of system (30) is invertible if and only if

$$
d \lambda_{k l} \neq p \text { and } d \lambda_{k l} \neq \frac{1}{p} \text { for }(k, l) \in[1, m] \times[1, n],
$$

where $\lambda_{k l}$ is defined by (51), $u_{i j}^{0}$ and $u_{i j}^{1}$ are 0 or $p-1$ for $[1, m] \times[1, n]$.

The second part of theorem is clear. The proof is complete.

Remark 3. In view of Lemma $2, W$ is an open set of $R^{m}$. Suppose that

$$
\min \left(\lambda_{12}, \lambda_{21}\right)>\frac{1}{d}+\frac{1}{d p}
$$

holds and

$$
d \lambda_{k l} \neq \frac{1}{p} \text { for }(k, l) \in[1, m] \times[1, n] .
$$

Then, the open set $W$ can be chosen the subset of $\left\{c \varphi_{i j}^{(k l)}, c \in R\right\}$, where $\varphi_{i j}^{(k l)}$ is defined in $(52),(i, j) \in[1, m] \times[1, n],(k, l) \in[2, m] \times[1, n]$ or $[1, m] \times[2, n]$. In this case, system (30) has $m n-1$ nontrivial positive solutions. On the other hand, if there exists $(k, l)$ and $\left(k^{\prime}, l^{\prime}\right)$ such that $\lambda_{k l}=\lambda_{k^{\prime} l^{\prime}}$, then the open set $W$ can also be chosen the subset of

$$
\left\{b \varphi_{i j}^{(k l)}+b \varphi_{i j}^{\left(k^{\prime} l^{\prime}\right)}, a, b \in R\right\} .
$$

Some new positive solutions will be added.

Remark 4. Certainly, the feedback control can be added when the system is unstable, see Shang [37]. Such work will be considered in the further.

\section{A simplest two elements system}

Motivated by the pattern formations of Section 3, we should further discuss the dynamical behaviors of (7)-(8). Unfortunately, we can not give a more general analysis. In this section, we will consider a simplest two elements system which is the case of $m=1$ and $n=2$, and hope to obtain some valuable informations. The simplest Neumann problem is

$$
\left\{\begin{array}{c}
u_{1}^{t+1}=d\left(-u_{1}^{t}+u_{2}^{t}\right)+\frac{p u_{1}^{t}}{1+u_{1}^{t}}, \\
u_{2}^{t+1}=d\left(u_{1}^{t}-u_{2}^{t}\right)+\frac{p u_{2}^{t}}{1+u_{2}^{t}} .
\end{array}\right.
$$

When $d=0$, system (33) is reduced to

$$
u^{t+1}=\frac{p u^{t}}{1+u^{t}}
$$

which has a unique positive steady state $p-1$ and is stable for $p>1$. 
Clearly, the point $E=(p-1, p-1)$ is also the unique positive equilibrium of (33). The linearization of (33) about $E$ has the Jacobian matrix

$$
J_{E}=\left(\begin{array}{cc}
-d+\frac{1}{p} & d \\
d & -d+\frac{1}{p}
\end{array}\right)
$$

which has the eigenvalues

$$
\lambda_{1}=\frac{1}{p}-2 d \text { and } \lambda_{2}=\frac{1}{p}
$$

and the corresponding eigenvectors

$$
\varphi^{(1)}=\operatorname{col}(-1,1) \text { and } \varphi^{(2)}=\operatorname{col}(1,1) .
$$

Note that $0<\lambda_{2}<1$ for $p>1$. We assume that $\lambda_{1}<-1$ and obtain that

$$
d>\frac{1+p}{2 p} .
$$

Property 1. For any $p>1$, the unique positive equilibrium $E=(p-1, p-1)$ of system (33) is stable if and only if

$$
d<\frac{1+p}{2 p} .
$$

In view of Property 1, we find that system (33) is Turing unstable if and only if

$$
d>\frac{1+p}{2 p} .
$$

Thus, $d=d_{1}=(1+p) / 2 p$ is the Turing unstable bifurcation of (33) and the Turing unstable domain is

$$
\Omega=\left\{(p, d): d>\frac{1+p}{2 p}, p>1\right\} .
$$

To obtain the further information, we let $d=d_{1}=(1+p) / 2 p$. In this case, we find that $\lambda_{1}=-1$ and $\lambda_{2}=1 / p<1$. Thus, $d=d_{1}=(1+p) / 2 p$ is also the flip bifurcation of (33).

Now, we assume that $d$ is a parameter and rewrite (33) as

$$
\left(\begin{array}{c}
u_{1} \\
u_{2} \\
d
\end{array}\right) \rightarrow\left(\begin{array}{c}
d\left(-u_{1}^{t}+u_{2}^{t}\right)+\frac{p u_{1}^{t}}{1+u_{1}^{t}} \\
d\left(u_{1}^{t}-u_{2}^{t}\right)+\frac{p u_{2}^{t}}{1+u_{2}^{t}} \\
d
\end{array}\right)
$$

Let

$$
x=u_{1}-u_{1}^{*}, y=u_{2}-u_{2}^{*} \text { and } \delta=d-d_{1} .
$$


We have

$$
\left(\begin{array}{l}
x \\
y \\
\delta
\end{array}\right) \rightarrow\left(\begin{array}{ccc}
\frac{1}{p}-d_{1} & d_{1} & 0 \\
d_{1} & \frac{1}{p}-d_{1} & 0 \\
0 & 0 & 1
\end{array}\right)\left(\begin{array}{l}
x \\
y \\
\delta
\end{array}\right)+\left(\begin{array}{c}
f_{1}(x, y, \delta) \\
g_{1}(x, y, \delta) \\
0
\end{array}\right)
$$

where

$$
\begin{gathered}
f_{1}=\frac{1}{2} a_{200} x^{2}+a_{101} x \delta+a_{011} y \delta+\frac{1}{6} a_{300} x^{3}+O(4), \\
g_{1}=\frac{1}{2} b_{020} y^{2}+b_{101} x \delta+b_{011} y \delta+\frac{1}{6} b_{030} y^{3}+O(4), \\
a_{100}=\frac{1}{p}-d_{1}, a_{010}=d_{1}, a_{200}=-\frac{2}{p^{2}}, a_{101}=-1, a_{011}=1, a_{300}=\frac{6}{p^{3}}, \\
b_{100}=d_{1}, b_{010}=\frac{1}{p}-d_{1}, b_{020}=-\frac{2}{p^{2}}, b_{010}=1, b_{011}=-1, b_{030}=\frac{6}{p^{3}}
\end{gathered}
$$

and $O(4)$ represents the terms with order greater than or equal to 4 in the variables $(x, y, \delta)$.

Assume that

$$
\left(\begin{array}{l}
x \\
y \\
\delta
\end{array}\right)=T\left(\begin{array}{l}
w \\
z \\
\delta
\end{array}\right)
$$

where

$$
T=\left(\begin{array}{ccc}
-1 & 1 & 0 \\
1 & 1 & 0 \\
0 & 0 & 1
\end{array}\right)
$$

which implies that

$$
T^{-1}=\left(\begin{array}{ccc}
-\frac{1}{2} & \frac{1}{2} & 0 \\
\frac{1}{2} & \frac{1}{2} & 0 \\
0 & 0 & 1
\end{array}\right)
$$

From (40), we get that

$$
\begin{aligned}
\left(\begin{array}{c}
w \\
z \\
\delta
\end{array}\right)= & T^{-1}\left(\begin{array}{ccc}
\frac{1}{p}-d_{1} & d_{1} & 0 \\
d_{1} & \frac{1}{p}-d_{1} & 0 \\
0 & 0 & 1
\end{array}\right) T\left(\begin{array}{c}
w \\
z \\
\delta
\end{array}\right) \\
& +T^{-1}\left(\begin{array}{c}
F_{1}(w, z, \delta) \\
G_{1}(w, z, \delta) \\
0
\end{array}\right) \\
= & \left(\begin{array}{ccc}
-1 & 0 & 0 \\
0 & \frac{1}{p} & 0 \\
0 & 0 & 1
\end{array}\right)\left(\begin{array}{c}
w \\
z \\
\delta
\end{array}\right)-\frac{1}{2}\left(\begin{array}{c}
F_{1} \\
G_{1} \\
0
\end{array}\right),
\end{aligned}
$$

where

$$
F_{1}=\frac{1}{p^{3}}\left(-p w^{2}-p z^{2}+2 p^{3} w \delta+2 p w z-w^{3}+z^{3}+3 w^{2} z-3 w z^{2}\right)
$$


and

$$
G_{1}=-\frac{1}{p^{3}}\left(p w^{2}+p z^{2}+2 p^{3} w \delta+2 p w z-w^{3}-z^{3}-3 w^{2} z-3 w z^{2}\right) .
$$

In the following, we will compute the center manifold $W^{c}(0,0,0)$. To this end, we assume that

$$
W^{c}(0,0,0)=\{(w, z, \delta): z=H(w, \delta), H(0,0)=0, D H(0,0)=0\},
$$

where

$$
H(w, \delta)=e_{1} w^{2}+e_{2} w \delta+e_{3} \delta^{2}+O(|w|+|\delta|)^{3} .
$$

Using the invariance of the center manifold, we get that

$$
e_{1}=\frac{1}{2(p-1)}, e_{2}=0 \text { and } e_{2}=0
$$

The map (41) restricted to the center manifold $W^{c}(0,0,0)$ is

$$
F: w \rightarrow-w+c_{20} w^{2}+c_{11} w \tau+c_{21} w^{2} \tau+c_{12} w \tau^{2}+c_{30} w^{3}+O(4),
$$

where

$$
c_{20}=c_{21}=c_{12}=0, c_{11}=-4, c_{30}=\frac{1-3 p}{(p-1) p^{3}} .
$$

Note that

$$
\eta_{1}=\left.\left(\frac{\partial^{2} F}{\partial w \partial \tau}+\frac{1}{2} \frac{\partial F}{\partial \tau} \frac{\partial^{2} F}{\partial w^{2}}\right)\right|_{(w, \tau)=(0,0)}=-4 \neq 0
$$

and

$$
\eta_{2}=\left.\left(\frac{1}{6} \frac{\partial^{3} F}{\partial w^{3}}+\left(\frac{1}{2} \frac{\partial^{2} F}{\partial w^{2}}\right)^{2}\right)\right|_{(w, \tau)=(0,0)}=\left(\frac{1-3 p}{(p-1) p^{3}}\right)^{2}>0 .
$$

In view of the flip bifurcation theorem in [11] or [27], we conclude the following result.

Theorem 3. For any $p>1$, system (33) undergoes a flip bifurcation and the bifurcated 2-periodic points are stable.

In the following, we will give a bifurcation diagram for $d>0$. For example, can we succeed when $p=2$ ? First of all, we randomly choose the initial values such that $\left(u_{1}^{0}, u_{2}^{0}\right) \in(1 \pm \varepsilon, 1 \pm \varepsilon)$. Unfortunately, all bifurcation diagrams are $u_{1}^{t} \equiv u_{2}^{t} \equiv 1$. By using the discrete Grobman-Hartman Theorem, also see Lemma 1 , for the fixed $p=2$, we choose that the initial values

$$
\operatorname{col}\left(u_{1}^{0}, u_{2}^{0}\right)=\operatorname{col}(1-\varepsilon, 1+\varepsilon),
$$

then the bifurcation diagrams will be obtained. For example, let $p=2$ and

$$
\operatorname{col}\left(u_{1}^{0}, u_{2}^{0}\right)=\operatorname{col}(1-0.001,1+0.001) .
$$


However, we also are defeated.

We find that our system (33) is very special. Once the computation is reached $u_{1}^{t_{0}}=u_{2}^{t_{0}}$ for some time $t_{0}$, the system will drop into the simple equation

$$
u_{1}^{t+1}=u_{2}^{t+1}=u^{t+1}=\frac{u^{t}}{1+u^{t}} \text { for } t \geq t_{0} .
$$

In this case, naturally, we have $\lim _{t \rightarrow \infty} u_{1}^{t}=\lim _{t \rightarrow \infty} u_{2}^{t}=\lim _{t \rightarrow \infty} u^{t}=1$. Thus, we need to avoid $u_{1}^{t_{0}}=u_{2}^{t_{0}}$ for some time $t_{0}$.

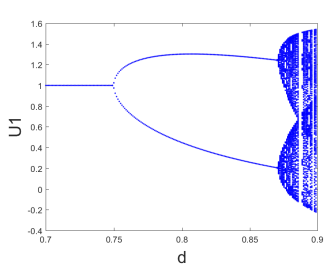

(a) $p=2$

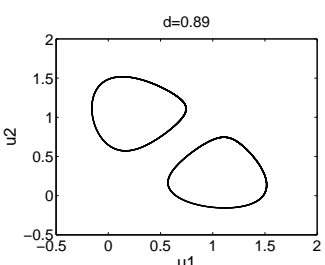

(b)

To overcome the above difficulties, we will use the shooting method. That is, once the computation is reached $u_{1}^{t_{0}}=u_{2}^{t_{0}}$ for some $t_{0}$, the computer will again compute from the initial values. It is very lucky, we obtain the following bifurcation diagram for $p=2$, please see (a) in Figure 4. When $p=2$ and $d \approx 0.89$, we also indeed obtain the other periodic solution, please see (b) in Figure 4.

\section{Conclusions and open problems}

In this paper, a single-handed discrete Fisher equation with the Neumann boundary conditions has been considered. By the linearization analysis, the necessary and sufficient condition of the diffusion-driven instability/Turing instability is obtained. In this case, a series of 2-periodic patterns have been obtained by the numerical simulations and the existence of 2-periodic solutions has also been established. Further results, a simplest two elements system has be discussed and the flip bifurcation theorem has been obtained by computing the center manifold. At the same time, the bifurcation diagrams will be simulated by using the shooting method. It proves that the Turing instability and the complexity of dynamical behaviors of (33) can be completely driven by the diffusion term. Finally, we provide some open problems.

Open Problem 1. Find the existence conditionds for other periodic solutions solutions of (7)-(8) and simulate the corresponding periodic patterns.

Open Problem 2. Design the efficient numerical methods for the bifurcation diagrams, the graghs of Lyapunov exponent, or the topological entropy of problem (7)-(8). 


\section{Acknowledgments}

The research is supported by the National Natural Science Foundation of China (No. 11371277). The authors are very thankful to the learned referees for valuable comments.

\section{References}

[1] F. T. Arecchi, S. Boccaletti and P. Ramazza, Pattern formation and competition in nonlinear optics, Physics Reports, 318(1999), 1-83.

[2] M. Barlo, G. Carmona and H. Sabourian, Bounded memory folk theorem, Journal of Economic Theory, 163(2016), 728-774.

[3] C. Castellano, S. Fortunato and V. Loreto, Statistical physics of social dynamics, Rev. Mod. Phys., 81(2007), 591-646.

[4] A. Blagodatski, et al., Diverse set of Turing nanopatterns coat cornea across insect lineages, PNAS, 112(2015), 10750-10755.

[5] Y. Cao, et al., Programmable assembly of pressure sensors using patternforming bacteria, Nature Biotechnology, 35(2017), 1087-1093.

[6] D. A. Christian, et al., Spotted vesicles, striped micelles and Janus assemblies induced by ligand binding, Nature Materials, 8(2009), 843-849.

[7] M. C. Cross and P. C. Hohenberg, Pattern formation outside equilibrium, Rev. Mod. Phys., 65(1993), 851-1112.

[8] H. I. Freedman, Deterministic Mathematical Models in Population Ecology, Marcel Dekker, New York, 1980.

[9] A. Gierer and H. Meinhardt, A theory of biological pattern formation, Kybernetik, 12(1972), 30-39.

[10] J. M. Grandmont, Nonlinear difference equations, bifurcations and chaos: An introduction, Research in Economics, 62(2008), 122-177.

[11] J. Guckenheimer and P. Holmes, Nonlinear Oscillations, Dynamical Systems, and Bifurcations of Vector Fields, Springer New York; 1983.

[12] Y. T. Han, et al., Turing instability and wave patterns for a symmetric discrete competitive Lotka-Volterra system, Wseas Transactions on Mathematics, 10(2011), 181-189.

[13] J. Huang, et al., Spontaneous formation of nanoparticle stripe patterns through dewetting, Nature Materials, 4(2005), 896-900.

[14] T. Huang and H. Zhang, Bifurcation, chaos and pattern formation in a space- and time-discrete predator-prey system, Chaos Soliton Fract., 91(2016), 92-107. 
[15] T. Huang, H. Zhang and H. Yang, Spatiotemporal complexity of a discrete space-time predator-prey system with self- and cross-diffusion, Applied Mathematical Modelling, 47(2017), 637-655.

[16] A. Jacobo and A. J. Hudspeth, Reaction-diffusion model of hair-bundle morphogenesis, PNAS, 111(2014), 15444-15449.

[17] A. Kicheva and J. Briscoe, Developmental pattern formation in phases, Trends in Cell Biology, 25(2015), 579-591.

[18] Yuri A. Kuznetsov, Elements of Applied Bifurcation Theory, SpringerVerlag, New York, 1998.

[19] M. S. Lavine, Turing structures at the nanoscale, Science, 360(2018), 503.

[20] K. J. Lee, et al., Pattern formation by interacting chemical fronts, Science, 261(1993), 192-194.

[21] Y. Lehahn, F. d'Ovidio, and I. Koren, A satellite-based lagrangian view on phytoplankton dynamics, Annual Review of Marine Science, 10(2018), 99-119.

[22] M. F. Li, et al., Spiral patterns near Turing instability in a discrete reaction diffusion system, Chaos, Solitons \& Fractals, 49(2013), 1-6.

[23] Y. Li, et al., Turing-type patterns on electrode surfaces, Science, 291(2001), 2395-2398.

[24] P. Z. Liu and X. Y. Cui, A discrete model of competition, Mathematics and Computers in Simulation, 49(1999), 1-12.

[25] L. A. Lugiato (Ed.), Nonlinear Optical Structures, Patterns and Chaos (special issue), Chaos, Solitons and Fractals, 4(8/9)(1994).

[26] R. M. May, Simple mathematical models with very complicated dynamics, Nature, 261(1976), 459-467.

[27] L. L. Meng, X. F. Li and G. Zhang, Simple diffusion can support the pitchfork, the flip bifurcations, and the chaos, Communications in Nonlinear Science and Numerical Simulation, Commun Nonlinear Sci Numer Simulat, 53(2017), 202-212.

[28] D. C. Mistro, LAD Eodrigues and S. Petrovskii, Spatiotemporal complexity of biological invasion in a space- and time-discrete predator-prey system with the strong Allee effect, Ecol Complex, 9(2012), 16-32.

[29] P. G. Moerman, et al., Emulsion patterns in the wake of a liquid-liquid phase separation front, PNAS, 115(2018), 3599-3604.

[30] J. D. Murry, Mathematical Biology, Springer-Verlag, New York, 1989. 
[31] M. Parsa, S. Harmand and K. Sefiane, Mechanisms of pattern formation from dried sessile drops, Advances in Colloid and Interface Science, 254(2018), 22-47.

[32] J. E. Pearson, Complex patterns in a simple system, Science, 261(1993), 189-192.

[33] D. Punithan, D. K Kim and R. McKay, Spatio-temporal dynamics and quantification of daisyworld in two-dimensional coupled map lattices, Ecological Complexity, 12(2012), 43-57.

[34] LAD Rodrigues, D. C. Mistro and S. Petrovskii, Pattern formation in a space- and time-discrete predator-prey system with a strong allee effect, Theor. Ecol. 5(2011), 341-62.

[35] Y. Shang, Modeling epidemic spread with awareness and heterogeneous transmission rates in networks, J. Biol. Phys., 39(2013), 489-500.

[36] Y. Shang, Discrete-time epidemic dynamics with awareness in random networks, International Journal of Biomathematics, 6(2)(2013), 1350007(7 pages), DOI: 10.1142/S1793524513500071.

[37] Y. Shang, Global stability of disease-free equilibria in a two-group SI model with feedback control, Nonlinear Analysis: Modelling and Control, $20(4)(2015), 501-508$.

[38] Y. L. Song, R. Yang and G. Q. Sun, Pattern dynamics in a GiererMeinhardt model with a saturating term, Applied Mathematical Modelling, 46(2017), 476-491.

[39] N. Stoop, et al., Curvature-induced symmetry breaking determines elastic surface patterns, Nature Materials, 14(2015), 337-342.

[40] G. Q. Sun, et al., Pattern transitions in spatial epidemics: Mechanisms and emergent properties, Physics of Life Reviews, 19(2016), 43-73.

[41] Z. Tan, et al., Polyamide membranes with nanoscale Turing structures for water purification, Science, 360(2018), 518-521.

[42] A. M. Tayar, et al., Synchrony and pattern formation of coupled genetic oscillators on a chip of artificial cells, PNAS, 114(2017), 11609-11614.

[43] N. Tompkins, et al., Testing Turing's theory of morphogenesis in chemical cells, PNAS, 111(2014), 4397-4402.

[44] A. M. Turing, The chemical basis of morphogenesis, Philos. Trans. Roy. Soc. London Ser. B, 237(1952), 37-72.

[45] J. L. Wang, Y. Li, S. H. Zhong and X. J. Hou, Analysis of bifurcation, chaos and pattern formation in a discrete time and space Gierer Meinhardt system, Chaos, Solitons and Fractals, 118(2019), 1-17. 
[46] Y. Wang, et al., Advanced colloidal lithography: From patterning to applications, Nano Today, 22(2018), 36-61.

[47] L. Xu, G. Zhang, H. Y. Cui, Dependence of initial value on pattern formation for a logistic coupled map lattice, Plos One, 11(2016): e0158591.

[48] L. Xu, et al., Turing instability for a two-dimensional Logistic coupled map lattice, Physics Letters A, 374(2010), 3447-3450.

[49] B. Yao, et al., Gate-tunable frequency combs in graphene-nitride microresonators, Nature, 558(2018), 410-414.

[50] W. C. Yueh, Eigenvalues of several tridiagonal matrices, Applied Math. E-Notes, 5(2005), 66-77.

[51] L. Zhang, G. Zhang and W. Feng, Turing instability generated from discrete diffusion-migeration systems, Canadian Applied Mathematics Quarterly, 20, Summer 2012, 253-269.

[52] G. Zhang, et al., Partial Difference Equation and Its Application, Science Press, Beijing, 2018(in Chinese).

\section{Appendix}

In the appendix, we will discuss the Neumann eigenvalue problems and the diffusion-driven instability or Turing instability of (7)-(8).

The discrete Neumann eigenvalue problem of the form

$$
\left\{\begin{array}{c}
-\Delta x_{i}=\lambda x_{i}, i \in[1, n], \\
x_{0}=x_{1}, x_{n}=x_{n+1}
\end{array}\right.
$$

had been considered in [50], also see [52], where $\Delta x_{i}=x_{i-1}-2 x_{i}+x_{i+1}$. Here, we will give other method.

For any $k \in[1, n]$, let

$$
\varphi_{i}^{(k)}=\cos \frac{(k-1)(2 i-1) \pi}{2 n}, i \in[1, n] .
$$

Note that

$$
\varphi_{0}^{(k)}=\cos \frac{-(k-1) \pi}{2 n}=\cos \frac{(k-1) \pi}{2 n}=\varphi_{1}^{(k)}
$$

and that

$$
\begin{aligned}
\varphi_{n}^{(k)} & =\cos \frac{(k-1)(2 n-1) \pi}{2 n} \\
& =\cos \left[(k-1) \pi-\frac{(k-1) \pi}{2 n}\right] \\
& =\cos \left[(k-1) \pi+\frac{(k-1) \pi}{2 n}\right] \\
& =\varphi_{n+1}^{(k)},
\end{aligned}
$$


thus, the function $\varphi_{i}^{(k)}$ satisfies the boundary conditions of (47). On the other hand, we have also

$$
\begin{aligned}
-\Delta \varphi_{i}^{(k)} & =-\left(\varphi_{i-1}^{(k)}-2 \varphi_{i}^{(k)}+\varphi_{i+1}^{(k)}\right) \\
& =4 \sin ^{2} \frac{(k-1) \pi}{2 n} \cos \frac{(k-1)(2 i-1) \pi}{2 n} \\
& =4 \sin ^{2} \frac{(k-1) \pi}{2 n} \varphi_{i}^{(k)} .
\end{aligned}
$$

Therefore, problem (47) has the eigenvalue

$$
\lambda_{k}=4 \sin ^{2} \frac{(k-1) \pi}{2 n}
$$

and the corresponding eigenvector

$$
\varphi_{i}^{(k)}=\cos \frac{(k-1)(2 i-1) \pi}{2 n}, i \in[1, n]
$$

for $k \in[1, n]$.

For $k, l \in[1, n]$ with $k \neq l$, we have

$$
\begin{aligned}
0 & =\sum_{i=1}^{n} \varphi_{i}^{(l)} \Delta \varphi_{i}^{(k)}+\lambda_{k} \sum_{i=1}^{n} \varphi_{i}^{(l)} \varphi_{i}^{(k)} \\
& =\left(\lambda_{k}-\lambda_{l}\right) \sum_{i=1}^{n} \varphi_{i}^{(l)} \varphi_{i}^{(k)}
\end{aligned}
$$

which implies that the inner product $\left(\varphi^{(k)}, \varphi^{(l)}\right)=0$ for $k \neq l$.

For the completeness, in the following, we will give unitization of the above vectors. To this end, let

$$
\beta=\frac{2(k-1) \pi}{n}
$$

Then

$$
\sin \left(i+\frac{1}{2}\right) \beta-\sin \left(i-\frac{1}{2}\right) \beta=2 \cos i \beta \sin \frac{\beta}{2}
$$

and

$$
\cos \left(i+\frac{1}{2}\right) \beta-\cos \left(i-\frac{1}{2}\right) \beta=-2 \sin i \beta \sin \frac{\beta}{2}
$$

or

$$
\cos i \beta=\frac{1}{2 \sin \frac{\beta}{2}}\left[\sin \left(i+\frac{1}{2}\right) \beta-\sin \left(i-\frac{1}{2}\right) \beta\right]
$$

and

$$
\sin i \beta=-\frac{1}{2 \sin \frac{\beta}{2}}\left[\cos \left(i+\frac{1}{2}\right) \beta-\cos \left(i-\frac{1}{2}\right) \beta\right] \text {. }
$$


Therefore, we have $\left\|\varphi^{(1)}\right\|^{2}=n$ and

$$
\begin{aligned}
& \left\|\varphi^{(k)}\right\|^{2}=\sum_{i=1}^{n} \cos ^{2} \frac{(k-1)(2 i-1) \pi}{2 n} \\
& =\frac{1}{2} \sum_{i=1}^{n}\left[1+\cos \frac{(k-1)(2 i-1) \pi}{n}\right] \\
& =\frac{n}{2}+\frac{1}{2} \cos \frac{\beta}{2} \sum_{i=1}^{n} \cos i \beta+\frac{1}{2} \sin \frac{\beta}{2} \sum_{i=1}^{n} \sin i \beta \\
& =\frac{n}{2}+\cos \frac{\beta}{2} \sum_{i=1}^{n} \frac{1}{4 \sin \frac{\beta}{2}}\left[\sin \left(i+\frac{1}{2}\right) \beta-\sin \left(i-\frac{1}{2}\right) \beta\right] \\
& -\sin \frac{\beta}{2} \sum_{i=1}^{n} \frac{1}{4 \sin \frac{\beta}{2}}\left[\cos \left(i+\frac{1}{2}\right) \beta-\cos \left(i-\frac{1}{2}\right) \beta\right] \\
& =\frac{n}{2}+\frac{\cos \frac{\beta}{2}}{4 \sin \frac{\beta}{2}}\left[\sin \left(n+\frac{1}{2}\right) \beta-\sin \frac{\beta}{2}\right] \\
& -\frac{1}{4}\left[\cos \left(n+\frac{1}{2}\right) \beta-\cos \frac{\beta}{2}\right] \\
& =\frac{n}{2}+\frac{\cos \frac{\beta}{2}}{4 \sin \frac{\beta}{2}} \sin \left(n+\frac{1}{2}\right) \beta-\frac{1}{4} \cos \left(n+\frac{1}{2}\right) \beta \\
& =\frac{n}{2}+\frac{\cos \frac{(k-1) \pi}{n}}{4 \sin \frac{(k-1) \pi}{n}} \sin \left[2(k-1) \pi+\frac{(k-1) \pi}{n}\right] \\
& -\frac{1}{4} \cos \left[2(k-1) \pi+\frac{(k-1) \pi}{n}\right] \\
& =\frac{n}{2} \text { for } k \in[2, n] \text {. }
\end{aligned}
$$

Now, the eigenvectors/eigenfunctions

$$
e_{i}^{(1)}=\frac{1}{\sqrt{n}} \varphi_{i}^{(1)} \text { and } e_{i}^{(k)}=\sqrt{\frac{2}{n}} \varphi_{i}^{(k)} \text { for } k \in[2, n] \text { and } i \in[1, n]
$$

form a standard orthogonal basis of (47).

In the following, we consider the discrete eigenvalue problem of the form

$$
\left\{\begin{array}{c}
-\Delta u_{i j}=\lambda u_{i j},(i, j) \in[1, m] \times[1, n] \\
u_{i, 0}=u_{i, 1}, u_{i, n}=u_{i, n+1}, i \in[0, m+1] \\
u_{0, j}=u_{1, j}, u_{m, j}=u_{m+1, j}, j \in[0, n+1]
\end{array}\right.
$$

where

$$
\Delta u_{i j}=u_{i+1, j}+u_{i, j+1}+u_{i-1, j}+u_{i, j-1}-4 u_{i j} .
$$


By using the separation variables method, we can obtain the eigenvalue

$$
\lambda_{k l}=4\left[\sin ^{2} \frac{(k-1) \pi}{2 m}+\sin ^{2} \frac{(l-1) \pi}{2 n}\right]
$$

and the corresponding eigenfunction

$$
\varphi_{i j}^{(k l)}=\cos \frac{(k-1)(2 i-1) \pi}{2 m} \cos \frac{(l-1)(2 j-1) \pi}{2 n},
$$

for $(i, j),(k, l) \in[1, m] \times[1, n]$.

Similarly, we can also obtain a standard orthogonal basis of (50) as follows

$$
\begin{gathered}
e_{i j}^{(11)}=\frac{1}{\sqrt{m n}} \phi_{i j}^{(11)}, \\
e_{i j}^{(k 1)}=\sqrt{\frac{2}{m n}} \phi_{i j}^{(k 1)} \text { for } k \in[2, m], \\
e_{i j}^{(1 l)}=\sqrt{\frac{2}{m n}} \phi_{i j}^{(1 l)} \text { for } l \in[2, n]
\end{gathered}
$$

and

$$
e_{i j}^{(k l)}=\sqrt{\frac{4}{m n}} \phi_{i j}^{(k l)} \text { for }(k, l) \in[2, m] \times[2, n] .
$$

